\title{
DETERMINAÇÃO POTENCIOMÉTRICA EM FLUXO DE CLORETO DE CETILPIRIDINIO EM DESINFECTANTES BUCAIS
}

Paula C. S. Baptista, Alberto N. Araújo e Maria da Conceição B. S. M. Montenegro*

CEQUP/Departamento de Química Física, Faculdade de Farmácia, Rua Aníbal Cunha, 164, 4050-047 Porto- Portugal

Recebido em 27/5/02; aceito em 10/12/02

\begin{abstract}
POTENTIOMETRIC DETERMINATION OF CETYLPYRIDINIUM CHLORIDE IN ORAL DESINFECTANTS BY FLOW INJECTION ANALYSIS. The work describes a new procedure for cetylpyridinium chloride determination in oral disinfectants, based on a flow-injection system with potentiometric detection. The determination was based on the measurement of picrate concentration decrease as result of ion-pair reaction with the analyte present in the injected sample. In the optimised set-up the sample injection volume was kept at $400 \mu \mathrm{L}$ and merged downstream with the reagent solution containing $1,0 \times 10^{-5} \mathrm{~mol} / \mathrm{L}$ of picrate adjusted to $\mathrm{pH} 5.0$ with citrate/citric acid buffer. The flow rate was fixed at $8 \mathrm{~mL} / \mathrm{min}$ and the reactor length at $40 \mathrm{~cm}$. The proposed procedure enables the determination of cetylpyridinium in the analytical range of $5,0 \times 10^{-6}-7,5 \times 10^{-5} \mathrm{~mol} / \mathrm{L}$ at a sampling rate of $60 / \mathrm{h}$. The results for real samples had a precision better than $3 \%$ and were comparable to the labelled values.
\end{abstract}

Keywords: potentiometry; oral disinfectants; FIA.

\section{INTRODUÇÃO}

Os surfactantes catiónicos encontram uma grande aplicação em diversas indústrias destacando-se a química (pesticidas), papel, têxtil, plásticos e tintas. Além disso, são frequentemente usados na preparação de produtos de limpeza doméstica e de higiene pessoal ${ }^{1}$ nomeadamente em desinfectantes bucais, pastas dentífricas, cremes de barbear, champôs e desodorizantes, por possuírem, para além de outras, propriedades anti-sépticas. É o caso do cloreto de cetilpiridinio, também designado por cloreto de hexadecilpiridinio que apresenta acção antimicrobiana, sobre bactérias gram negativas e que frequentemente é utilizado em fórmulas desinfectantes bucais. Embora sob o ponto de vista ambiental a sua determinação seja menos importante que a dos surfactantes aniónicos ${ }^{2}$ é conveniente o seu controlo analítico nos produtos comerciais referidos.

Para a determinação de surfactantes catiónicos são descritos diversos procedimentos baseados na utilização de métodos volumétricos ${ }^{3,4}$ espectrofotométricos ${ }^{5-7}$, $\operatorname{cromatográficos}^{8-10}$, electroforese capilar ${ }^{11,12}$ e potenciométricos ${ }^{13-25}$. Estes últimos, aplicados de forma geral à determinação indirecta de surfactantes catiónicos em soluções desinfectantes oferecem como vantagens a determinação rápida, a preparação simples das amostras, a elevada sensibilidade e um grau de selectividade que conduz à obtenção de resultados mais exactos ${ }^{26,27}$.

O desempenho analítico dos eléctrodos selectivos de iões, poderá ainda ser incrementado recorrendo a métodos de fluxo contínuo, em particular à técnica Análise por Injecção em Fluxo (FIA). A eliminação da solução de referência interna dos eléctrodos tem possibilitado a sua construção com configuração tubular e incorporação em montagens FIA simples, fisicamente robustas, resultando em algumas situações a um aumento de reprodutibilidade das medidas ${ }^{28}$ e da sensibilidade analítica $(\mathrm{mV} / \mathrm{dec})^{29}$ e mais frequentemente a diminuição das interferências ${ }^{30}$. Estes aspectos são vantajosos sob o ponto de vista do controlo de rotina em laboratórios de produção industrial.

Procurando aproveitar as vantagens da potenciometria com eléctrodos selectivos de ião como processo de detecção em FIA esta-

*e-mail: mcbranco@ff.up.pt beleceu-se uma metodologia simples para a análise do cloreto de cetilpiridinio em desinfectantes bucais vulgarmente disponíveis no comércio.

A determinação baseava-se na reacção entre o cloreto de cetilpiridinio e o picrato, com formação de um complexo iónico, sendo posteriormente o picrato remanescente monitorado potenciometricamente. Para isso utilizou-se um detector, de configuração tubular, sensível aquele anião, construído segundo a metodologia proposta anteriormente ${ }^{31}$.

\section{PARTE EXPERIMENTAL}

\section{Reagentes e soluções}

Para a preparação das soluções usou-se água bidesionizada com condutância especifica igual ou inferior a $0,1 \mu \mathrm{S} / \mathrm{cm}$. Todos os reagentes usados eram de qualidade analítica ou semelhante, sem terem sido submetidos a qualquer purificação adicional.

A solução "stock" de picrato de sódio, com concentração igual a $6,0 \times 10^{-2} \mathrm{~mol} / \mathrm{L}$, foi obtida a partir de uma solução de ácido pícrico (Merck, ref. 623), com igual concentração, previamente titulada com hidróxido de sódio até $\mathrm{pH}$ 6,0, garantindo a desprotonação total do ácido pícrico. As soluções padrão de picrato de sódio foram obtidas a partir da anterior, por diluição com sulfato de sódio $3,3 \times 10^{-2} \mathrm{~mol} / \mathrm{L}$, por forma a ajustar a força iónica a um valor igual a $0,1 \mathrm{~mol} / \mathrm{L}$. Estas soluções foram usadas na preparação do sensor iónico das membranas dos eléctrodos e na avaliação das características de funcionamento das unidades construídas, em condições de fluxo. Na selecção da solução reagente usada no procedimento desenvolvido utilizaram-se soluções de picrato de sódio preparadas em tampão ácido cítrico/citrato de sódio com pH igual a 5,0.

As soluções de referência de cloreto de cetilpiridinio (Fluka, ref. 52349) usadas na calibração do procedimento proposto foram preparadas por diluição de uma solução $1,0 \times 10^{-2} \mathrm{~mol} / \mathrm{L}$. As diluições foram efectuadas com água bidesionizada.

As membranas dos eléctrodos foram preparadas com cloreto de bis(trifenilfosforanilideno)amónio (Aldrich, ref. 22383-2), 2nitrofeniloctiléter (Fluka, ref. 73732) e PVC (Fluka, ref. 81431). 
As amostras de produtos comerciais analisados, obtidas de modo aleatório no comércio local, foram sujeitas a diluição apropriada $(1: 100 \mathrm{v} / \mathrm{v})$ com água desionizada antes da sua introdução no sistema FIA.

\section{Material e equipamento utilizado na montagem FIA}

Na montagem FIA estabelecida (Figura 1), as soluções eram impulsionadas através de uma bomba peristáltica, de quatro canais Gilson, modelo Minipuls 2 (Gilson ${ }^{\circledR}$ Inc., Villiers-le-Bel, França) usando-se tubos de impulsão de PVC da mesma marca, com diâmetro interno de $2 \mathrm{~mm}$. As soluções padrão e as amostras eram inseridas no fluxo transportador através de uma válvula de injecção rotatória manual Rheodyne, modelo 5020 (Rheodyne, São Francisco, EUA). $\mathrm{O}$ volume de amostra injectado foi definido pelo comprimento do tubo de PTFE com 0,8 mm de diâmetro interno, usado como alça de amostragem. As ligações entre os diferentes componentes da montagem FIA foram efectuadas usando tubos de PTFE, com 0,8 mm de diâmetro interno, munidos de terminais e ligadores da marca Gilson. Para promover uma mistura adequada entre a amostra e a solução de picrato, o reactor $\mathrm{L}_{1}$ foi enrolado, em forma de oito, sobre uma rede plástica, com uma altura de aproximadamente $2 \mathrm{~cm}$. Para promover a confluência das soluções usou-se uma peça com um percurso tubular gravado em forma de $\mathrm{Y}$, com diâmetro interno de $0,8 \mathrm{~mm}$. A célula potenciométrica era constituída por um eléctrodo indicador de configuração tubular, sensível ao anião picrato associado a um eléctrodo de referência de dupla junção, da marca Orion, modelo 90-00-29 (Thermo Orion, Beverly, EUA) que continha no compartimento externo sulfato de sódio $3,3 \times 10^{-2} \mathrm{~mol} / \mathrm{L}$. A escolha desta solução, decorreu da baixa interferência do anião sulfato no comportamento dos eléctrodos sensíveis a picrato ${ }^{31}$ Para reduzir o ruído eléctrico induzido pelo sistema de propulsão foi inserido no sistema FIA um eléctrodo de terra construído de acordo com o descrito na literatu$\mathrm{ra}^{32}$. Os suportes para os referidos eléctrodos foram construídos em poli(metacrilato de metilo) ${ }^{33}$ de modo a possibilitarem a sua colocação no sistema numa posição fixa. A medida da diferença de potencial entre os eléctrodos indicador e de referência foi efectuada com um decimilivoltímetro da marca Crison, com impedância de entrada superior a $12 \mathrm{M} \Omega$, modelo MicropH 2002 (Crison, Barcelona, Espanha) acoplado a um registador Metrohm, Labograph, modelo E-586 (Metrohm Ltd., Herisau, Suiça).

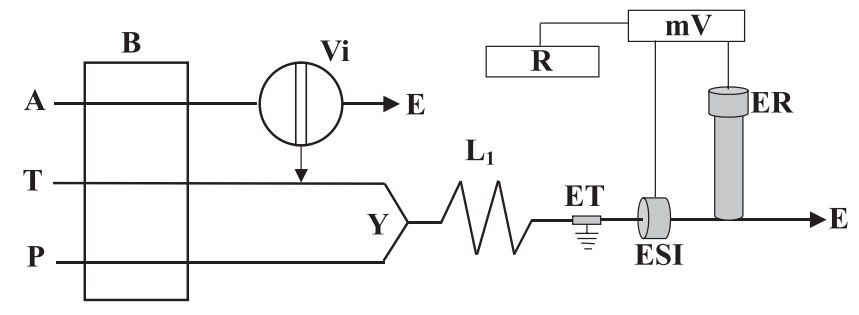

Figura 1. Esquema da montagem FIA desenvolvida para a determinação de cloreto de cetilpiridinio com detecção potenciométrica. $B$ - bomba peristáltica; $\mathrm{Vi}$ - volume de injecção; A - amostra; $\mathrm{T}$ - solução transportadora $\left(\mathrm{Na}_{2} \mathrm{SO}_{4}\right.$ $\left.3,3 \times 10^{-2} \mathrm{~mol} / \mathrm{L}\right) ; P$ - picrato tamponado; $Y$ - confluência; $L_{1}$ - reactor; $E T$ eléctrodo de terra; ESI - eléctrodo selectivo ao anião picrato; ER-eléctrodo de referência; $E$ - esgoto; $m V$-decimilivoltímetro; $R$ - registador

\section{Construção dos eléctrodos tubulares sensíveis ao anião picrato}

Os eléctrodos sensíveis ao anião picrato foram construídos segundo o procedimento descrito anteriormente por Lopes et al. ${ }^{31}$. O sensor iónico resultou da reacção estequiométrica que decorreu da adição a uma solução de picrato de sódio $1,0 \times 10^{-3} \mathrm{~mol} / \mathrm{L}$, de igual volume de uma solução aquosa de extractor iónico, o cloreto de bis(trifenilfosforaniliden)amónio, na mesma concentração. Os cristais obtidos (picrato de bis(trifenilfosforanilideno) amónio), foram lavados com água bi-desionizada e guardados em frasco de vidro escuro no excicador. Após a sua secagem, dissolveram-se 0,0431 g dos cristais em 0,7886 g de 2-nitrofeniloctiléter (solvente mediador). Esta solução sensora foi imobilizada em PVC (0,18 g) previamente dissolvido em tetrahidrofurano e foi posteriomente depositada no orifício central do eléctrodo tubular ${ }^{33}$. As membranas dos eléctrodos eram secas à temperatura ambiente durante $24 \mathrm{~h}$. O seu condicionamento era efectuado com os eléctrodos inseridos na montagem FIA, fazendo-se passar a um caudal de cerca de $0,2 \mathrm{~mL} / \mathrm{min}$,

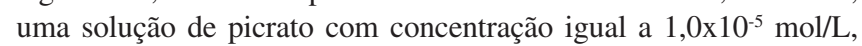
durante cerca de $2 \mathrm{~h}$.

\section{RESULTADOS E DISCUSSÃO}

Antes de proceder ao desenvolvimento e optimização de uma montagem de fluxo que permitisse a análise de cloreto de cetilpiridinio em fórmulas comerciais, avaliou-se a resposta dos detectores potenciométricos construídos no sistema FIA de baixa dispersão. Para o efeito, procedeu-se ao traçado de curvas de calibração, utilizando soluções com concentração crescente em picrato de sódio. $\mathrm{O}$ volume de injecção seleccionado foi de $200 \mu \mathrm{L}$ e usou-se um fluxo transportador de sulfato de sódio $3,3 \times 10^{-2} \mathrm{~mol} / \mathrm{L}$ que continha picrato de sódio em concentração igual a $1,0 \times 10^{-6} \mathrm{~mol} / \mathrm{L}$ para estabilizar a linha de base. O transportador fluía a um caudal de $6 \mathrm{~mL} / \mathrm{min}$, dirigindo-se para o detector potenciométrico colocado a uma distância de $40 \mathrm{~cm}$ do ponto de injecção. Nestas condições, a resposta dos eléctrodos era linear no intervalo de concentrações entre $1,0 \times 10^{-6} \mathrm{e}$ $1,0 \times 10^{-3} \mathrm{~mol} / \mathrm{L}$, com um declive de $-54,5 \pm 0,3 \mathrm{mV} /$ déc e um coeficiente de correlação quadrático igual a 0,998 .

Para viabilizar a determinação do surfactante em estudo, estabeleceu-se uma montagem FIA com a configuração representada na Figura 1. Nesse sistema, uma alíquota da amostra era injectada na solução de transporte de sulfato de sódio, com concentração igual a $3,3 \times 10^{-2} \mathrm{~mol} / \mathrm{L}$, misturando-se de seguida em Y com uma solução de picrato de sódio de concentração e $\mathrm{pH}$ conhecidos e fixados. Ao longo do reactor $\mathrm{L}_{1}$ ocorria a formação do par iónico entre o cloreto de cetilpiridinio e o picrato, sendo a diminuição da concentração deste anião quantificada através do eléctrodo tubular.

Os diferentes parâmetros da montagem estabelecida foram optimizados pelo método univariado, de modo a obter o melhor compromisso entre sensibilidade, reprodutibilidade, repetibilidade, frquência de amostragem e economia de reagentes. Para optimizar a concentração do reagente utilizaram-se diferentes soluções de picrato, preparadas em solução $3,3 \times 10^{-2} \mathrm{~mol} / \mathrm{L}$ de $\mathrm{Na}_{2} \mathrm{SO}_{4}$, com concentrações do anião que variaram entre $1,0 \times 10^{-6}$ e $1,0 \times 10^{-4} \mathrm{~mol} / \mathrm{L}$. Este estudo foi realizado fixando o volume de injecção em $200 \mu \mathrm{L}$, a concentração de cloreto de cetilpiridinio em $5,0 \times 10^{-5} \mathrm{~mol} / \mathrm{L}$, o comprimento do reactor de mistura $\mathrm{L}_{1}$ em $40 \mathrm{~cm}$ e o caudal a $4 \mathrm{~mL} / \mathrm{min}$. Nestas condições a intensidade do sinal analítico obtido aumentou de cerca de $32 \pm 1$ para $47 \pm 1 \mathrm{mV}$ quando a concentração de picrato variou entre $1,0 \times 10^{-6} \mathrm{~mol} / \mathrm{L} \mathrm{e} 1,0 \times 10^{-5} \mathrm{~mol} / \mathrm{L}$, não se registando alterações na intensidade dos sinais obtidos para as concentrações mais elevadas do reagente. $\mathrm{O}$ efeito do $\mathrm{pH}$ na extensão da reacção de complexação foi avaliado para soluções de picrato com concentração igual a $1,0 \times 10^{-5} \mathrm{~mol} / \mathrm{L}$, preparadas em tampões com $\mathrm{pH}$ de 4,0; 5,0; 7,0; 7,5 e 9,0. Para ajustar o pH a 4,0; 5,0; e 7,0 usou-se tampão de ácido cítrico/citrato de sódio; enquanto para os valores 7,5 e 9,0 recorreu-se ao tampão Tris/HCl. Os resultados obtidos (Figura 2) 
demonstram um máximo de intensidade do sinal analítico para o valor de pH igual a 5,0. Após a fixação destes parâmetros, estudouse a resposta do detector potenciométrico para caudais que variavam entre 3 e $12 \mathrm{~mL} / \mathrm{min}$ (Figura 3) registando-se um aumento da intensidade dos sinais analíticos com o aumento do caudal até se atingir um patamar no intervalo entre $8-10 \mathrm{~mL} / \mathrm{min}$. Optou-se por fixar o valor deste parâmetro em $8 \mathrm{~mL} / \mathrm{min}$ por ser o melhor compromisso entre frequência de amostragem e sensibilidade. Por outro lado, verificou-se que caudais superiores a $8 \mathrm{~mL} / \mathrm{min}$, conduziam a um aumento da irreprodutibilidade dos sinais analíticos. Seguidamente, foi efectuado o estudo da influência do volume de injecção na amplitude do sinal analítico quando se seleccionavam valores entre 100 e $750 \mu \mathrm{L}$. Os resultados obtidos evidenciaram uma diminuição da dispersão da amostra com o aumento do volume injectado. A altura do sinal analítico crescia até um valor máximo e igual a $400 \mu \mathrm{L}$ estabilizando posteriormente o que justificou a escolha deste valor nos ensaios posteriores. O comprimento e a forma de enrolamento do reactor $\mathrm{L}_{1}$, situado entre a confluência $\mathrm{Y}$ e o detector, foram seleccionados de modo a facilitar a mistura entre a solução reagente e a amostra, sem contudo introduzir um apreciável incremento da dispersão do produto de reacção,verificando-se que para o comprimento de $60 \mathrm{~cm}$ se observava o melhor compromisso entre sensibilidade e frequência de amostragem (Figura 4). Efetuaram-se curvas de calibração com soluções de referência de cloreto de cetilpiridinio, cuja concentração variava entre $5,0 \times 10^{-6}$ e $1,0 \times 10^{-4} \mathrm{~mol} / \mathrm{L}$ evidenciando-se que o procedimento proposto permitia determinações numa gama de concentrações entre $5,0 \times 10^{-6}$ e $7,5 \times 10^{-5} \mathrm{~mol} / \mathrm{L}$, com um con-

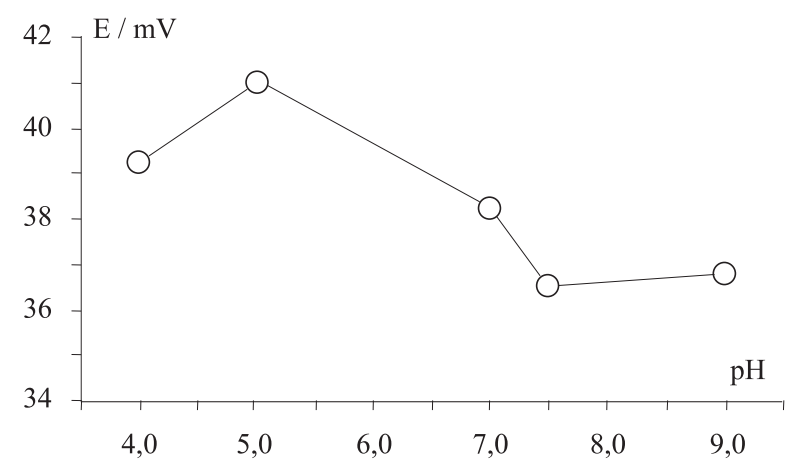

Figura 2. Variação da amplitude do sinal analítico em função do $p H$ de soluções com concentração de picrato igual a $1 \times 10^{-5} \mathrm{~mol} / \mathrm{L}$. A solução injectada continha cloreto de cetilpiridinio na concentração de $1 \times 10^{-4} \mathrm{~mol} / \mathrm{L}$

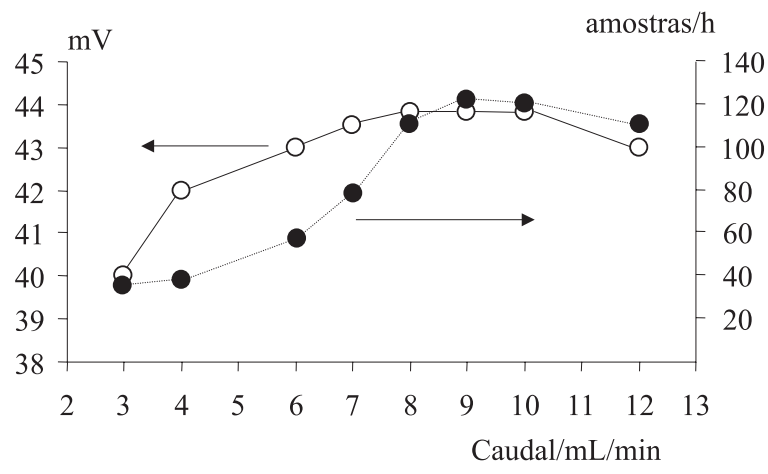

Figura 3. Influência do caudal na intensidade do sinal analítico e na frequência de amostragem. Condições iguais às fixadas na figura anterior sumo de reagente de $18,3 \mu \mathrm{g}$ por medida, com um coeficiente de correlação quadrático $\left(\mathrm{R}^{2}\right)$ de 0,996 e com uma frequência analítica correspondente a 60 amostras $/ \mathrm{h}$. Sendo um processo de determinação indirecto, a reduzida faixa analítica obtida comparativamente com o que é característico da potenciometria directa com eléctrodos selectivos de iões é condicionada pela concentração de picrato no transportador que proporciona maior sensibilidade para baixas concentrações do analito.

\section{ANÁLISE DE AMOSTRAS REAIS}

Com o sistema de fluxo desenvolvido efetuaram-se análises em amostras de desinfectantes bucais, nomeadamente Oral B, Aquafresh, Reach e Denthal, comercializadas em Portugal. A Figura 5 representa um fiagrama típico obtido da análise de um dos produtos e na Tabela 1 apresentam-se os resultados referentes à média de 3 determinações sobre a mesma amostra. Para efeitos de comparação e por não existir nenhum método oficial aplicado à análise deste tipo de produtos, incluem-se igualmente na Tabela 1 os correspondentes valores indicados no rótulo pelos fabricantes. Relativamente aos excipientes das amostras analisadas, cuja indicação era fornecida pelo fabricante, não foram registadas interferências significativas . Os ensaios de recuperação efectuados por adição às amostras diluídas de um volume igual de soluções de cloreto de cetilpiridínio com

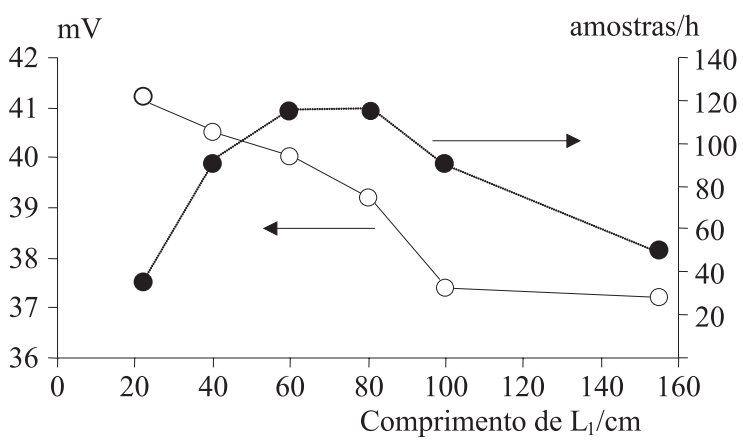

Figura 4. Influência do comprimento do reactor $L_{1}$ na intensidade do sinal analítico e na frequência de amostragem, quando era disposto rectilineamente. Condições iguais às fixadas na Figura 2

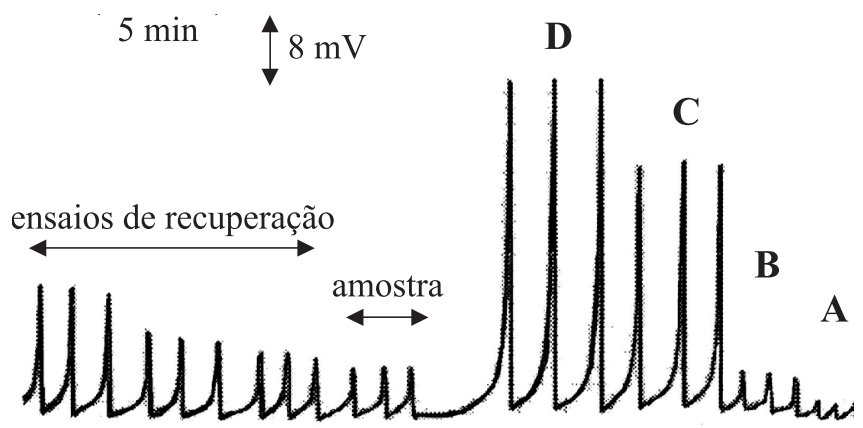

Figura 5. Fiagrama correspondente à injecção de uma série de padrões de cloreto de cetilpiridinio, com concentrações: $A-5,0 \times 10^{-6} ; B-1,0 \times 10^{-5}$; $C-5,0 \times 10^{-5} ; D-7,5 \times 10^{-5} \mathrm{~mol} / \mathrm{L}$, e à análise de uma solução desinfectante bucal ( $A_{2}$-Aquafresh) e do ensaio de recuperação, correspondente a três adições de padrões de cloreto de cetilpiridinio nas concentrações de, respectivamente, $5,0 \times 10^{-6} \mathrm{~mol} / \mathrm{L}, 7,5 \times 10^{-6} \mathrm{~mol} / \mathrm{L}$ e $1,0^{\prime} 10^{-5} \mathrm{~mol} / \mathrm{L}$ 
Tabela 1. Resultados da determinação de cloreto de cetilpiridinio em desinfectantes bucais pelo procedimento proposto. Os resultados correspondem à média de três determinações

\begin{tabular}{lcc}
\hline Amostras & $\begin{array}{c}\text { Valor indicado no } \\
\text { rótulo }(\mathrm{mol} / \mathrm{L})\end{array}$ & $\begin{array}{c}\text { Valor obtido } \\
(\mathrm{mol} / \mathrm{L})\end{array}$ \\
\hline Oral B & $1,39 \times 10^{-3}$ & $(1,10 \pm 0,03) \times 10^{-3}$ \\
Aquafresh & $1,39 \times 10^{-3}$ & $(1,10 \pm 0,03) \times 10^{-3}$ \\
Reach & $1,39 \times 10^{-3}$ & $(1,19 \pm 0,01) \times 10^{-3}$ \\
Deenthal & $0,70 \times 10^{-3}$ & $(0,76 \pm 0,01) \times 10^{-3}$ \\
\hline
\end{tabular}

concentrações dentro da faixa linear revelaram em todos os casos a obtenção de valores de recuperação médios de $98 \pm 3 \%$. Estes resultados permitiram confirmar a boa exactidão do procedimento proposto, aspecto que confirma o bom desempenho analítico da potenciometria já evidenciado anteriormente por outros autores ${ }^{24,25}$.

\section{CONCLUSÕES}

O controlo de surfactantes, nomeadamente o cloreto de cetilpiridinio em amostras comerciais é possível recorrendo a um método simples baseado na técnica de injecção em fluxo com detecção potenciométrica. O sistema proposto que é simples de operar e de fácil recurso a qualquer laboratório da indústria, apresenta como principal vantagem a possibilidade de fornecer resultados com boa reprodutibilidade a uma elevada frequência de amostragem e com um baixo consumo de reagentes. Os resultados sugerem a possibilidade de se aplicar uma montagem deste tipo à análise de outros surfactantes em amostras do tipo semelhante, nomeadamente, detergentes, sabões e champôs.

\section{REFERÊNCIAS}

1. Connell, D. W.; Basic Concepts of Environmental Chemistr, Lewis Publishers: USA, 1997, p. 221.

2. Luigi, C.; Lorenzo, A.; Claudio, C.; Mauro, T.; Anal. Lett. 1997, 30, 161.
3. Yamamoto, K.; Sakuno, K.; Bunseki Kagaku 1997, 46, 653.

4. Uemura, Y. H. K.; Ishihara, S.; Analyst 1994, 119, 473.

5. Kawase, J.; Yamanaka, M.; Analyst 1979, 104, 750.

6. Kawase, J.; Anal. Chem. 1980, 52, 2124.

7. Simon, M. M. B.; Cozar, A. E.; Diez, L. M. P.; Analyst 1990, 115, 337.

8. Nitschke, L. ; Muelles, R.; Metzner, L.; Huber, L.; Fresenius'J. Anal. Chem. 1992, 342, 711 .

9. Gort, S. M.; Hogendoorn, E. A.; Baumann, R. A.; Van Zoonen, P.; Int. J. Environ. Anal. Chem. 1993, 53, 289.

10. Brenn, D. G. P. A.; Horner, J. M.; Bartle, K. D.; Cliford, A. A.; Water, J.; Larwience, J. G. ; Anal. Chem. 1995, 67, 4210.

11. Heinig, K.; Vogt, C.; Werner, G.; J. Chromatogr. 1997, 781, 17.

12. Heinig, K.; Vogt, C.; Werner; G.; J. Chromatogr. 1997, 358, 500.

13. Selig, W.; Anal. Lett. 1982, 15 (A3), 309.

14. Matysik, S.; Matysik, F. M.; Mattusch, J.; Einicke, W. D.; Electroanalysis 1998, 10, 98 .

15. Metrohom Application Note, T-13, 2001, Pp.1.

16. Buschmann, N.; Schulz, R.; Tenside, Surfactants, Deterg. 1993, 29, 128.

17. Buschmann, N.; Schulz, R.; Tenside, Surfactants, Deterg. 1993, $30,18$.

18. Shoukry, A. F.; Badawy, S. S.; Farghali, R. A.; Anal. Chem. 1988, 60, 2399.

19. Campanella, L.; Aiello, L.; Colapiochioni, C.; Tomasseti, M.; Analusis 1996, 24, 387.

20. Egorov, V. V.; Repin, V. A.; Kaputskii, V. E.; J. Anal. Chem. 1996, 51, 986.

21. Vytras, K.; Microchem. Acta III 1984, 1-2, 139.

22. Vytras, K.; Kalous, J.; Symersky, J.; Anal. Chim. Acta 1985, 177, 219.

23. Abbas, M. N.; Mostafa, G. A. E.; Homoda, A. M. A.; Talanta 2000, 53, 425.

24. Tyagi, V.; Jain, A. K.; Indian J. Chem., Sect. A: Inorg., Bio-inorg., Phys., Theor. Anal. Chem. 1991, 30, 844.

25. Mostafa, G. A. E.; Anal. Sci. 2001, 17, 1043.

26. Zhang, Z. Z.; Cosofret, V. V.; Electrode. Rev. 1990, 12, 35.

27. Cosofret, V. V.; Buck, R. P.; Crit. Rev. Anal. Chem. 1993, 24, 1.

28. Lima, J. L. F. C.; Montenegro, M. C. B. S. M.; Sales, M. G. F.; J. Pharm. Sci. 1997, 86, 1234.

29. Lima, J. L. F. C.; Montenegro, M. C. B. S. M.; Sales, M. G. F.; J. Pharm. Biomed. Anal. 1996, 14, 931.

30. Lima, J. L. F. C.; Montenegro, M. C. B. S. M.; Silva, A. M. R.; J. Pharm. Biomed. Anal. 1991, 9,1041.

31. Lopes, T. I. M. S.; Rangel, A. O. S. S.; Lima, J. L. F. C; Montenegro, M. C. B. S. M.; Anal. Chim. Acta 1995, 308, 122.

32. Alegret, S.; Alonso, J.; Bartroli, J.; Machado, A. A. S. C.; Lima, J. L. F. C.; Paulis, J. M.; Quim. Anal. 1987, 6, 278.

33. Lima, J. L. F. C.; Montenegro, M. C. B. S. M.; Silva, A. M. R.; J. Flow Injection Anal. 1990, 7, 19. 